\title{
Mind the message: Referral letter quality at a South African medical outpatient department
}

\author{
I L Haeusler, ${ }^{1,2}$ MA, BM BCh, MRCPCH, DTM\&H; M Sajan, ${ }^{3}$ BPT, MSc; A Parrish, ${ }^{3,4}$ FCP (SA), MMedSci (Clinical Epidemiology) \\ ${ }^{1}$ Thames Valley and Wessex Leadership Academy, National Health Service, UK \\ ${ }^{2}$ Institute of Child Health, University College London, UK \\ ${ }^{3}$ Cecilia Makiwane Hospital, Mdanstsane, South Africa \\ ${ }^{4}$ Department of Internal Medicine, Faculty of Health Sciences, Walter Sisulu University, Mthatha, South Africa
}

Corresponding author: I L Haeusler(ilsa.haeusler@gmail.com)

\begin{abstract}
Background. Some patients need referral within the health system to achieve optimal care, and referral letters are an important part of this process. Healthcare practitioners often complain that referral letters lack information, are inaccurate, or direct patients to the wrong place. Poor communication affects patient experience and outcomes, has budgetary and service planning implications, and impacts on staff relationships and morale.

Objectives. To investigate the quality and appropriateness of referral letters received by the medical outpatient department of a regional hospital in Eastern Cape Province, South Africa.

Methods. Letters were collected by departmental staff as patients arrived at the clinic. Each letter was independently analysed by two healthcare workers for content and appropriateness, using defined criteria.

Results. Of 100 letters collected between March and May 2017, 85 were suitable for analysis. Patient and clinician identifiers were present in $>85 \%$, but key clinical information was missing in $87 \%$, and $48 \%$ did not state a reason for referral. It was possible to make triage decisions based on the letter in only $35 \%$ of cases. Nineteen percent of referrals were classified as inappropriate.

Conclusions. Most letters lacked important clinical information, probably because of a combination of factors: gaps in clinical knowledge of referring clinicians who service a population with a high burden of disease and complex pathology; under-resourced peripheral healthcare clinics; inadequate staff-to-patient ratios; and time constraints. A suggested focus for improvement is education at undergraduate and postgraduate level, which should emphasise preparation for community service, specifically highlighting techniques for preparing goodquality referrals.
\end{abstract}

S Afr Med J 2020;110(5):396-399. https://doi.org/10.7196/SAMJ.2020.v110i5.14275

For patients to receive appropriate care, it is often necessary to transfer them between healthcare facilities. A referral is a request to take over or give input into patient management, and involves communication between colleagues. ${ }^{[1]}$ Information may be transmitted verbally (in person or telephonically), or in a written referral letter. A good referral letter contains an adequately detailed summary of presentation, diagnostic process and current management, as well as an indication of why further clinical input or transfer of care is requested.

Anecdotal evidence and published reports suggest that clinicians often complain that referrals are unnecessary, are directed to the wrong site or arrive at an inappropriate time, and that referral letters are of poor quality. ${ }^{[2]}$ Letters may not provide sufficient accurate information to enable the recipient to take over care or provide useful advice. ${ }^{[3]}$ Such problems are bidirectional, ${ }^{[4]}$ and may reflect the frustrations of busy clinicians or indicate a deeper systematic problem of poor working relationships between healthcare providers. Perceptions of inaccurately or incompletely portrayed clinical situations, inappropriate referrals, or resistance to accept referrals can lead to feelings of resentment. This reaction may reduce collaborative potential and impact negatively on care.

Assessing referral letter quality is subjective. ${ }^{[5]}$ Most studies that have attempted it are from well-resourced healthcare systems. ${ }^{[3,4]}$ Under-resourced systems may have a different structure, less access to information technology, and a different burden of disease. A higher proportion of care is often provided by nurses or community health workers in such settings. ${ }^{[6-9]}$

\section{Objectives}

To assess the quality and appropriateness of a sample of written referrals to the medical outpatient department (OPD) of a regional hospital in Eastern Cape Province, South Africa (SA).

\section{Methods}

Setting

This prospective observational study was carried out in the medical OPD at Cecilia Makiwane Hospital (CMH), a 650-bed governmentfunded regional hospital in Mdanstsane, Eastern Cape. The referral area incorporates 21 district hospitals, a community health centre and 11 municipal clinics, and the medical OPD sees $\sim 17000$ patients annually, of whom 3800 (22\%) are new referrals (hospital data). The healthcare needs of the population served are high; life expectancy of men and women in the Eastern Cape is 58.5 and 65.9 years, respectively, ${ }^{[10]}$ and the prevalence of HIV infection was $26.9 \%$ in a recent serosurvey study of patients attending the emergency department at a nearby hospital. ${ }^{[1]}$ Eighty-eight percent of the population of the Eastern Cape rely on the public health sector for healthcare. ${ }^{[12]}$

The organisation of the public healthcare system is complex, with patients entering and moving around the system at different points for different types of care. ${ }^{[13]}$ Primary healthcare clinics are typically the entry point. Further community services, including homebased care, HIV voluntary counselling and testing, and tuberculosis treatment monitoring, are provided by community health workers. 
Referral to care at district hospitals, regional hospitals (such as $\mathrm{CMH}$ ) and then tertiary hospitals occurs when more specialised healthcare is required.

Healthcare workers at peripheral clinics and hospitals may initially refer by telephone, and a date on which the patient should attend is provided. The date is usually immediate (same day) or the next available date if the case is not perceived as urgent or if there are transport problems. The patient is given a referral letter, which is passed to clinic staff on arrival at $\mathrm{CMH}$. Patients are then triaged, and it is not uncommon for patients to be admitted directly from the OPD to the inpatient wards.

\section{Procedure}

The nursing staff collected and photocopied referral letters at the time of patient arrival in the OPD, with the aim of collecting 100 consecutive letters. Letters from healthcare practitioners other than medical doctors (such as physiotherapists and dieticians) and those that were addressed to facilities other than $\mathrm{CMH}$ were excluded. Referrals directed from tertiary clinics solely requesting specific follow-up investigations for sharedcare patients were also excluded.

\section{Variables}

Through discussions with the medical team and review of previous literature, a set of criteria were established that were considered to constitute elements of an appropriate and high-quality referral (for the variables, see Supplementary Material 1, available at http:// www.samj.org.za/public/sup/14275.pdf). It was agreed that a good-quality referral letter should at least contain patient and referring doctor details, information about the clinical problem, and the reason for referral. In the literature, there are no validated tools to assess referral letter quality, and each study available uses different combinations of variables to assess quality. Although an SA national referral template is occasionally used by some district hospitals in the referral region of $\mathrm{CMH}$, this document is difficult to source publicly. Apart from the 'ability to triage' variable, each of the other variables collected in this study represents components of the SA national referral template.

Data from each letter were independently extracted by two healthcare workers. The first group of variables were extracted by a senior physiotherapist (MS) and a junior doctor (ILH). The second group of variables were extracted by two medical doctors (ILH and $\mathrm{AP}$ ), because these variables were more clinical in nature. Data were anonymised and entered into an Excel spreadsheet, 2002 version (Microsoft, USA), and apart from the quantification of clinical information, each variable was binary (yes or no). Discrepancies were discussed between the two team members extracting the same data, and if consensus was not reached, the third team member was consulted for a consensus.

\section{Ethics approval}

Although there was no direct patient involvement, written ethics approval was obtained according to the institutional policy of the East London Hospitals Complex Ethics Review Board.

\section{Results}

The 100 referral letters were collected between 20 March and 11 May 2017. They represented $\sim 23 \%$ of new patients who were referred to the medical OPD at $\mathrm{CMH}$ during this period (based on hospital data). The nursing staff reported that they were unable to collect every letter owing to time constraints in a busy clinical environment; there was no reason to suspect selection bias, but this could not be specifically excluded in the absence of an analysable denominator. In total, 15 letters were excluded and 85 were retained for analysis, with the reasons for exclusion given in Fig. 1. All letters were handwritten.

\section{Administrative variables}

Table 1 displays the proportion of letters that included each administrative variable. The majority of letters contained the name of the referring hospital, the first name and surname of the patient, and the referring doctor's surname. Letters were dated in $86 \%$ of cases, and the referring doctor's telephone contact details were included in $47 \%$. The SA national referral template was used for $13 \%$ of letters.

\section{Clinical variables}

Every letter contained clinical information (Table 2). One letter contained fewer than 10 words describing the clinical problem, $88 \%$ had between 11 and 75 words, and $11 \%$ had more than 76 words. A clear clinical summary or differential diagnosis was not provided in $29 \%$ of letters, and $48 \%$ did not state a reason why the patient needed to be referred. The information provided was inconsistent in $42 \%$ of letters (the summary of clinical findings, differentials or required next steps did not follow logically from the clinical history or examination findings described).

Chronic medications with doses were detailed in $18 \%$ of letters. Only $13 \%$ of letters contained the necessary relevant clinical information from which a patient could be managed further. From the information provided in the letters, $35 \%$ of patients were able to be triaged (an assessment of how urgently a patient needs to be reviewed, particularly identifying those who required immediate attention). Referrals were appropriate in $81 \%$ of cases.

\section{Discussion}

Although there may be many ways to write a good referral letter, at its core it should deliver a concise summary of relevant information. Judgement of the contents of referral letters is inherently subjective. It is difficult to assess quality on the basis of the letter alone without seeing the patient; a judgement is made about the referrer's

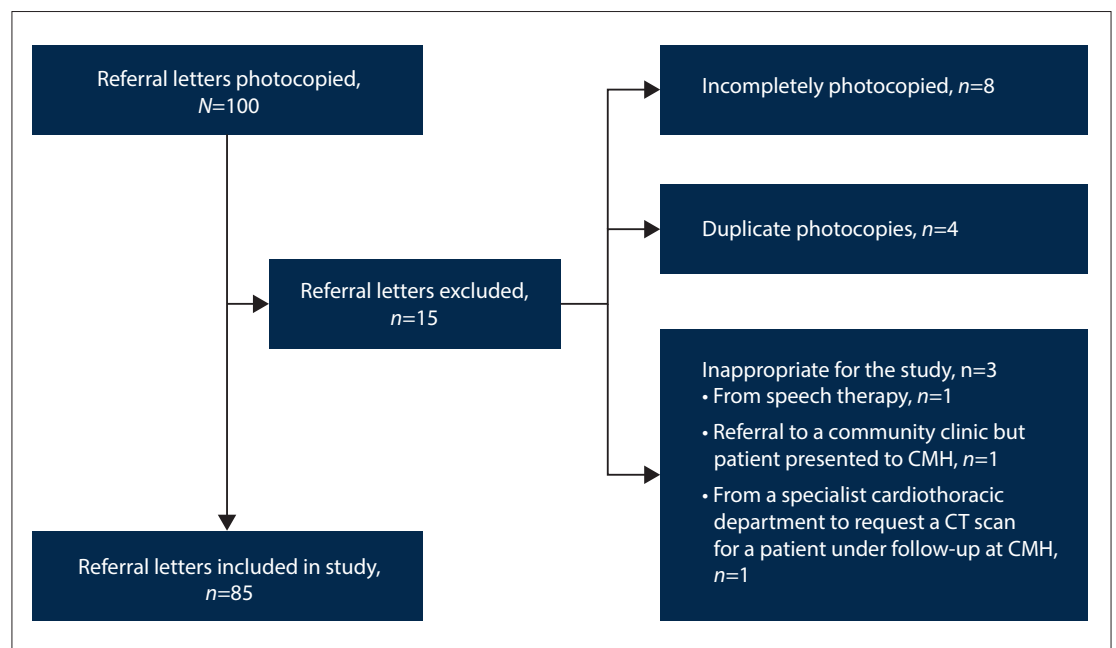

Fig. 1. Flow diagram of referral letter inclusion. $(C M H=$ Cecilia Makiwane Hospital; $C T=$ computed tomography.) 
Table 1. Summary of administrative variables ( $N=85$ letters)

\begin{tabular}{ll}
\hline Variable & 'Yes', $\boldsymbol{n}(\%)$ \\
\hline Name of referring hospital present? & $79(93)$ \\
Patient's first and surname present? & $78(92)$ \\
Referring doctor's surname present? & $74(87)$ \\
Letter dated? & $73(86)$ \\
Doctor's contact telephone present? & $40(47)$ \\
National template used? & $11(13)$
\end{tabular}

Table 2. Summary of clinical variables ( $N=85$ letters)

\begin{tabular}{ll}
\hline Variable & 'Yes', $\boldsymbol{n}$ (\%) \\
\hline Any clinical information present? & $85(100)$ \\
Referral appropriate? & $69(81)$ \\
Summary assessment, differential diagnosis? & $60(71)$ \\
Is the information consistent? & $49(58)$ \\
Reason stated for referral? & $44(52)$ \\
Can the patient be triaged? & $30(35)$ \\
Chronic medications with doses noted? & $15(18)$ \\
All relevant clinical information included? & $11(13)$ \\
&
\end{tabular}

documentation rather than the accuracy of the interpretation of clinical information. We assessed quality using multiple variables to get a sense of which variable or combination of variables was most helpful to objectively assess quality.

This study of the quality of referrals received by a medical OPD in the Eastern Cape, SA, showed that only $13 \%$ contained adequate clinical information and nearly half did not state why the patient needed to be referred.

These results are in line with other published studies of referral quality. ${ }^{[3]}$ While substantially more evidence has been obtained from high-income settings, ${ }^{[14]}$ some work has been carried out in SA. A literature review published in 1998 found two SA studies on the quality of referrals to secondary care. ${ }^{[15]}$ In one of these studies, similar methods were used to collect and analyse referral letters to specialist paediatric care in SA, and found that details of history and diagnosis were present in only $52.1 \%$ and $76.9 \%$ of letters, respectively. ${ }^{[16]}$ In the other study, 74\% of letters contained no history. ${ }^{[17]}$ In 1996, a study in KwaZulu-Natal Province found that the introduction of a referral pro forma improved the quality of the information contained in letters from general practitioners. ${ }^{[18]}$

More recently, a study from Gauteng Province published in 2011 used similar criteria to assess referral quality, and found that the doctor's demographic details were present in $96.8 \%$ of letters, with $80 \%$ dated and $80 \%$ detailing the main complaint, but only $25 \%$ containing past medical history. ${ }^{[19]}$

Similar work has been undertaken in other low- and middleincome countries. A Nigerian study assessed the quality of referrals to the psychiatry department of a teaching hospital. ${ }^{[20]}$ The clinical impression was included in $75 \%$ of letters, but medications were documented in only $16.5 \%$. In a survey undertaken in six Latin American countries, fewer than half of the specialist doctors surveyed stated that they regularly received information on clinical history, treatment or tests performed. ${ }^{[21]}$

The way information is communicated is influenced by personal style and enthusiasm. These characteristics, as well as the variability of clinical information that can be included, are reasons why it is difficult to align letters to one accepted format. Constructing (and receiving) letters that, in different ways, communicate urgency, importance, uncertainty or fascination is, for many people, part of the joy and 'art' of medicine. For this reason, it may not be desirable to try to standardise every aspect of referral documentation.

The ability to triage based only on the letter itself, possible in only $35 \%$ of our cases, was helpful in giving an overall impression of the clinical situation. In the context of an under-resourced, busy clinical environment, it is important to quickly formulate an impression of how unwell the patient is and how urgently they need to be seen. Common methods used to indicate urgency included listing vital signs and describing emergency clinical signs.

In $42 \%$ of letters the clinical information was incoherent or inconsistent, resulting in difficulty formulating an overall understanding of the clinical problem. Such difficulty causes frustration that can develop into mistrust of the information presented. A technique to improve clarity is to include a summary assessment, impression or differential diagnosis, which were present in only $29 \%$ of letters.

The two-page national referral template, which was used in only $13 \%$ of our cases, is organised into sections requesting the same type of information that was collected as variables in this study, and that can provide useful prompts if used and completed correctly. The main constraint of forms with boxes of fixed size is that they may limit the ability to include information, or even suggest that little information is required in an area particularly important for an individual patient.

Referrals are not unidirectional; the flow of information back to the referring team is as important as the initial referral. It allows the clinical team to understand the management plan. It also provides an opportunity for professional development through seeing the referral outcome. Without this bidirectional flow of information, the information gained during the referral consultation is lost.

The deficiencies we identified in the quality and appropriateness of referrals reveal broader problems in the SA healthcare system. The organisation of the healthcare system is difficult for both patients and healthcare providers to navigate (anecdotal evidence from experience and conversations with colleagues and patients). Moving between facilities is costly and leads to lengthy delays in clinical care as a result of large distances between clinics and insufficient patient transport services.

Much clinical care is provided by community service doctors with few years of clinical experience and working with limited senior supervision. Classic medical education focuses on the practice of medicine in a context with ready availability of senior colleagues, and where decision-making is less influenced by resource constraints. Although medical schools prepare doctors for community service and rural work, techniques to manage patients safely and efficiently with fewer resources and less help warrant emphasis, and one component of this is the ability to communicate well with colleagues. The importance of the 'soft skills' required to foster this culture of collaborative care should be embedded in the curriculum and become an examinable component of exit assessment.

\section{Study limitations}

There is no gold standard to assess referral letters, and few recent studies in a similar context have attempted this. There are other aspects of referral that were not examined (specifically, whether even poor letters may represent good clinical decisions), and the small sample size may not allow generalisation.

Referral appropriateness is difficult to judge, particularly without seeing and examining each patient. Referrals that could have been 
managed locally but gave the impression that the clinical team did not understand the problem (through incoherent or incomplete information) were judged as inappropriate.

\section{Conclusions}

Referral letters are a common source of frustration for clinicians. This study found that clinical information was inconsistent in nearly half of letters received by a referral facility in SA, with the majority of letters missing at least some important clinical information. Specific emphasis should be placed on preparation of medical students and junior clinicians for community service in district facilities with limited supervision and resources. One teachable and examinable skill is the ability to communicate with colleagues accurately and efficiently when transferring patients or seeking advice. Clinicians should foster an atmosphere of collaborative care, where amicable and efficient communication will enhance patient outcomes and experience.

\section{Declaration. None.}

Acknowledgements. We are very grateful to the nursing staff for collecting referral letters, and to Dr Jenny Nash for providing invaluable expertise on the local referral system.

Author contributions. AP oversaw concept development. ILH, MS and AP designed the study and collected data. ILH undertook data analysis. ILH wrote the first draft of the paper; MS and AP provided further input on subsequent versions.

Funding. No specific funding was received for this work. The Improving Global Health Fellowship (held by ILH) was funded by the National Health Service Thames Valley and Wessex Leadership Academy.

Conflicts of interest. None.

\footnotetext{
1. World Health Organization. Management of health facilities: Referral systems. Overview of referral
} systems. https://www.who.int/management/facility/referral/en/ (accessed 19 May 2019).
2. Martinussen PE. Referral quality and the cooperation between hospital physicians and general practice: The role of physician and primary care factors. Scand J Public Health 2013;41(8):874-882. https://doi.
. The role of physician and prim

3. Vermeir P, Vandijck D, Degroote S, et al. Communication in healthcare: A narrative review of the 3. Vermeir P, Vandijck D, Degroote S, et al. Communication in healthcare: A narrative review of the
literature and practical recommendations. Int J Clin Pract 2015;69(11):1257-1267. https://doi. org/10.1111/ijcp.12686

4. Kripalani S, LeFevre F, Phillips CO, Williams MV, Basaviah P, Baker DW. Deficits in communication and information transfer between hospital-based and primary care physicians: Implications for patient safety and continuity of care. JAMA 2007;297(8):831-841. https://doi.org/10.1001/jama.297.8.831

5. Garåsen H, Johnsen R. The quality of communication about older patients between hospital physicians and general practitioners: A panel study assessment. BMC Health Serv Res 2007;7:133. https://doi. org/10.1186/1472-6963-7-133

6. Akbari A, Mayhew A, Al-Alawi MA, et al. Interventions to improve outpatient referrals from primary care to secondary care. Cochrane Database Syst Rev 2005, Issue 3. Art. No.: CD005471. https://doi. org/10.1002/14651858.CD005471

7. Corwin P, Bolter T. The effects of audit and feedback and electronic referrals on the quality of primary care referral letters. J Prim Health Care 2014;6(4):324-327.

8. Tattersall MH, Butow PN, Brown JE, Thompson JF. Improving doctors' letters. Med J Aust 2002;177(9):516-520. https://doi.org/10.5694/j.1326-5377.2002.tb04926.x

9. Wahlberg H, Valle PC, Malm S, Broderstad AR. Impact of referral templates on the quality of referrals from primary to secondary care: A cluster randomised trial. BMC Health Serv Res 2015;15:353. https:// doi.org/10.1186/s12913-015-1017-7

10. Statistics South Africa. Mid-year population estimates. 2018. http://www.statssa.gov.za/publications/ P0302/P03022018.pdf (accessed 19 May 2019)

11. Hansoti B, Stead D, Eisenberg A, et al. A window into the HIV epidemic from a South African emergency department. AIDS Res Hum Retroviruses 2018;35(2):139-144. https://doi.org/10.1089/aid.2018.0127

12. Eastern Cape Department of Health, South Africa. http://www.echealth.gov.za/index.php (accessed 19 May 2019).

13. African Institute of Health and Leadership Development. The minimum data sets for human resources for health and the surgical workforce in South Africass health system: A rapid analysis of stock and migration. 2015. https://www.who.int/workforcealliance/031616south_africa_case_studiesweb.pdf (accessed 19 May 2019).

14. Dinsdale E, Hannigan A, O'Connor R, et al. Communication between primary and secondary care: Dinsdale E, Hannigan A, O'Connor R, et al. Communication between primary and seron
Deficits and danger. Fam Pract 2020;37(1):63-68. https://doi.org/10.1093/fampra/cmz037

15. Couper I. Referral letters and replies. S Afr Fam Pract 1998;19(3).

16. Lachman PI, Stander IA. The referral letter - a problem of communication. S Afr Med J 1991;79(1):98-100.

17. Meiring JH, van den Berg AD. [By his letters shall the doctor be known]. S Afr Med J 1989;75(1):26-28

18. Couper ID, Henbest RJ. The quality and relationship of referral and reply letters: The effect of introducing a pro forma letter. S Afr Med J 1996;86(12):1540-1542.

19. Langalibalele M, Maduna P, Mhlongo S, Ndimande J, Longo-Mbenza B. Quality of general practitioner referral letters to a South African tertiary hospital: Determinants of quality content and good practice. J Public Health Epidemiol 2011;3(11):482-488.

20. Esan O, Oladele O. Referral letters to the psychiatrist in Nigeria: Is communication adequate? Afr Health Sci 2016;16(4):1023-1026. https://doi.org/10.4314/ahs.v16i4.19

21. Vargas I, Garcia-Subirats I, Mogollón-Pérez AS, et al. Understanding communication breakdown in the outpatient referral process in Latin America: A cross-sectional study on the use of clinical correspondence in public healthcare networks of six countries. Health Policy Plan 2018;33(4):494-504. https://doi.org/10.1093/heapol/czy016

Accepted 1 October 2019. 